ORIGINAL PAPER

\title{
INVASIVE DUCTAL CARCINOMA OF NO SPECIAL TYPE AND ITS \\ CORRESPONDING LYMPH NODE METASTASIS: DO THEY HAVE THE SAME IMMUNOPHENOTYPIC PROFILE?
}

\author{
Veaceslav Fulga ${ }^{1}$, Lucian Rudico ${ }^{1}$, Amalia Raluca Balica ${ }^{2}$, Anca Maria Cimpean ${ }^{2}$, \\ Lilian Saptefrati ${ }^{1}$, Marius Raica ${ }^{2}$
}

\begin{abstract}
${ }^{1}$ Department of Histology, Cytology and Embryology, State Medical University "Nicolae Testemitanu”, Chisinau, Republic of Moldova

2Department of Microscopic Morphology/Histology, Angiogenesis Research Center, "Victor Babes” University of Medicine and Pharmacy, Timisoara, Romania
\end{abstract}

\begin{abstract}
In the present study we compared the immunophenotypic subtypes of breast ductal invasive carcinomas with their ipsilateral, axillary lymph node metastasis. The ER (estrogen receptor), PR (progesterone receptor), Her2 (human epidermal growth factor receptor 2), and CK5 (cytokeratin 5) status and the proliferation marker Ki-67 were determined by immunohistochemistry on specimens from 43 women. All selected cases were diagnosed as invasive breast carcinomas, of no special type (NST), G2 grade of differentiation. The most frequently encountered subtype at both sites was luminal B. We determined that tumor profile evaluated by surrogate markers is not stable during the metastatic process. The total rate of shifted cases was $23.26 \%$ (10 cases), and the highest rate of shifting $(6.98 \%)$ was encountered from luminal $\mathrm{B} / \mathrm{Ki}-67$ to luminal A subtype. In five cases, the subtype shifted to a poorer one according to prognosis. These data support the hypothesis that breast cancer is a heterogeneous disease, with substantial variability of cellular components within each category, a statement applicable to invasive breast carcinomas of NST type too. The receptor profile of this carcinoma, indicated by surrogate markers, is not stable throughout the metastatic process.
\end{abstract}

Key words: breast cancer, metastasis, surrogate markers.

\section{Introduction}

Breast cancer is the most common cancer in women worldwide and one of the malignant diseases most studied by scientific communities [1]. It is described as a heterogeneous disease, variable in its clinical course, pathological aspects, therapy and prognosis $[2,3]$. Breast tumors with similar histology may express various clinical and pathological features. Because the previous classifications focusing only on morphology could not fully capture the diversity of the disease, another classification system, based on hormone receptor status and gene expression profile of breast cancers, has been developed [4, 5]. Nowadays, estrogen receptor (ER), progesterone receptor (PR) and Her2 receptor status of breast tumors can be determined by using immunohistochemical markers as surrogates, and the breast cancer cases can be classified into at least four molecular subtypes including luminal A, luminal B, basal-like and Her2/neu.

An important decisional factor of therapeutic strategy, which determines the patients' future outcome, is the presence of metastasis, especially in the regional 
lymph nodes. Traditionally, the presence of metastasis and the number and level of involved lymph nodes were described. Nowadays, it seems to be important to investigate the molecular profile of metastasis. Some data reveal the instability of tumor cell receptors throughout the metastatic process [6-10]. But few results concerning this field are published and existing data are quite sparse. One reason could be the inhomogeneous groups for analysis. Because invasive ductal cancer is one of the most frequent diagnoses in histopathological practice, we considered it opportune to describe this type of cancer in relation to its lymph node metastasis from the molecular classification position. Based on Goldhirsch's recommendations for oncological practice, the intrinsic subtypes have been defined by five surrogate markers [11]. In the present work we found that the molecular profile of invasive breast cancer of no special type is not homogeneous and not stable during tumorigenesis.

\section{Material and methods}

\section{Patient data}

In this retrospective study, we used the specimens from 43 women who underwent a radical mastectomy and lymph nodes dissection in the Oncological
Institute, Republic of Moldova, between 2012-2013. Patients did not receive radio- and/or chemotherapy before surgery. The ages of the women ranged from 37 to 85 years old. Three independent pathologists confirmed histopathology of the tumors. Histological grade was scored by the Scarff-Bloom-Richardson grading system, with Ellston-Ellis modification. All 43 selected cases were diagnosed as invasive ductal carcinoma of NST type and G2 grade of differentiation (as the most commonly encountered cases in the studied group).

\section{Tissue processing}

The specimens were fixed in $10 \%$ phosphate buffered formalin and paraffin embedded. Sections were sectioned and stained with hematoxylin (Mayer) and eosin (HE) for routine histopathological assessment. To avoid any misunderstandings about tissue processing, primary tumor and lymph node from the same patient were embedded in a single paraffin block and sections were stained on the same slide (Figs. 1, 2). The lymph node metastases were confirmed by immunohistochemistry with an AE1/ AE3 cytokeratin cocktail. In addition, sections were also stained with 5 monoclonal antibodies (Table I). All stages of immunohistochemistry, from dewax to
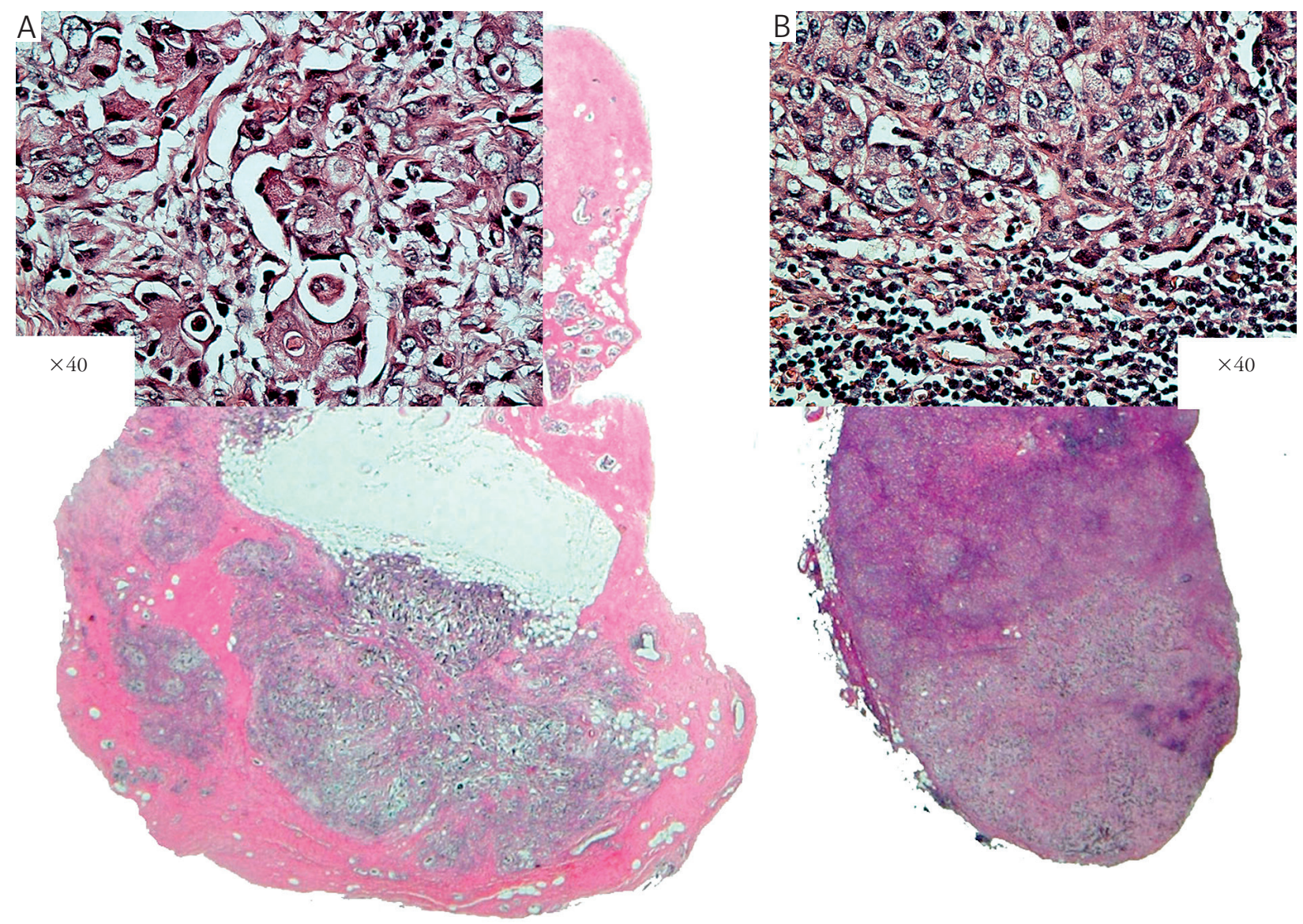

Fig. 1. Breast carcinoma NST type. Primary tumor (A) and its LNM (B) processed on the same slide. HE, magnification $10 \times$ 
A
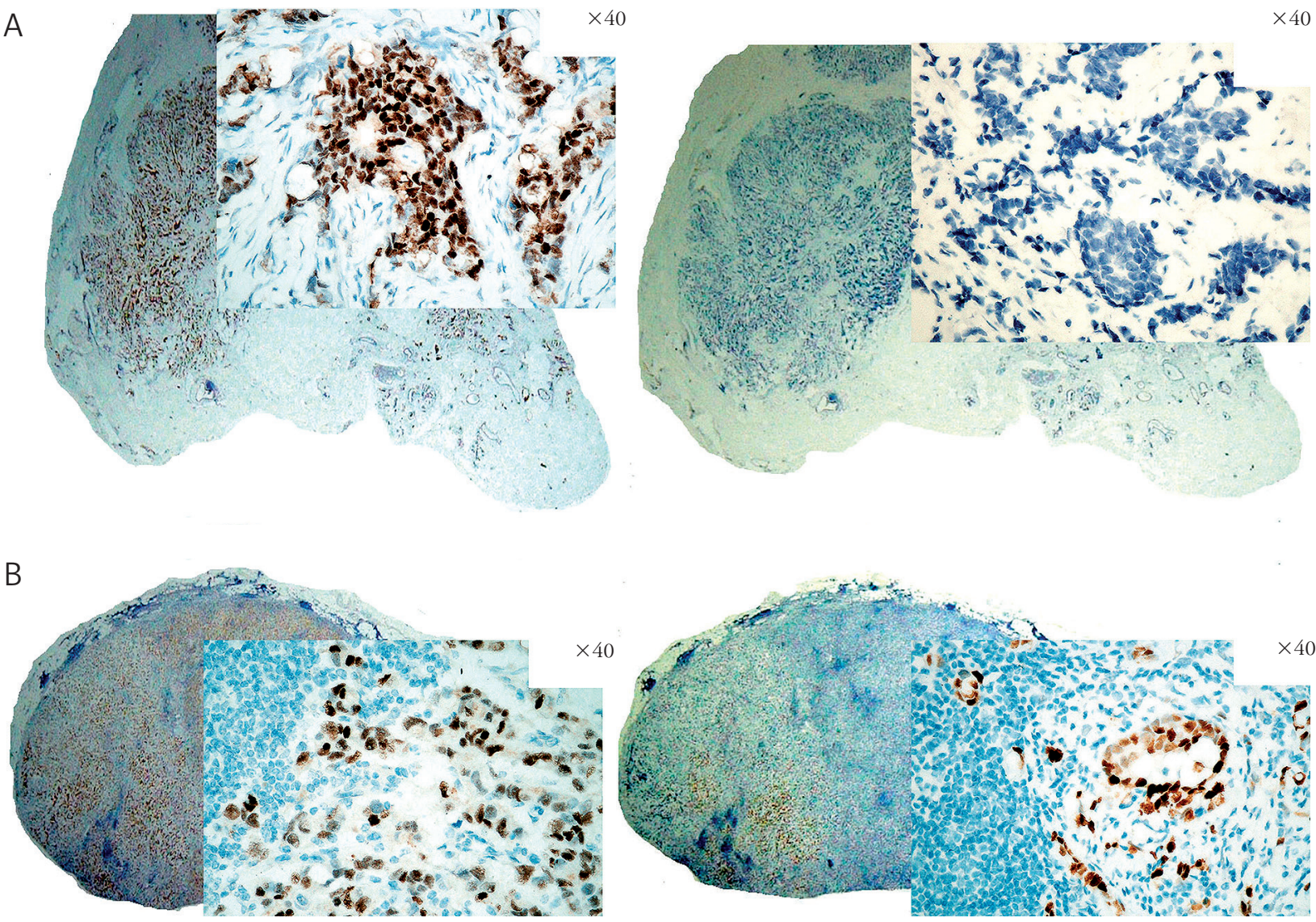

Fig. 2. Breast carcinoma NST type. Primary tumor (A) and its LNM (B) stained with anti-ER (I) and anti-PR (II) markers, magnification $10 \times$

Table I. Antibodies and conditions used for immunohistochemical analysis

\begin{tabular}{|c|c|c|c|}
\hline ANTIBODY/CLONE & $\begin{array}{c}\text { SOURCE/INCUBATION TIME/ } \\
\text { DILUTION }\end{array}$ & RETRIEVAL SYSTEM/TIME & DeteCtion/TIME \\
\hline $\mathrm{ER} / 6 \mathrm{~F} 11$ & \multirow{3}{*}{$\begin{array}{c}\text { Leica Biosystem Newcastle } \\
\text { Ltd, Newcastle Upon Tyne, } \\
\text { UK/15 min/RTU }\end{array}$} & \multirow{3}{*}{$\begin{array}{l}\text { Bond Epitope Retrieval } \\
\text { Solution 1, (Leica } \\
\text { Biosystems, Newcastle } \\
\text { Upon Tyne, UK)/20 min }\end{array}$} & \multirow{3}{*}{$\begin{array}{c}\text { Bond Polymer Refine } \\
\text { Detection System (Leica } \\
\text { Biosystems, Newcastle } \\
\text { Upon Tyne, UK), } 15 \text { min }\end{array}$} \\
\hline $\mathrm{PR} / 16$ & & & \\
\hline Multi-cytokeratin/(AE1/AE3) & & & \\
\hline Her2 /policlonal & $\begin{array}{c}\text { Dako Glostrup } \\
\text { Denmark/30 min/RTU }\end{array}$ & $\begin{array}{l}\text { Dako Target Retrieval } \\
\text { Solution, pH6/20 min }\end{array}$ & EnVision-HER/30 min \\
\hline $\mathrm{Ki}-67 / \mathrm{K} 2$ & $\begin{array}{c}\text { Leica Biosystem Newcastle } \\
\text { Ltd, Newcastle Upon Tyne, } \\
\text { UK/15 min/RTU }\end{array}$ & \multirow{2}{*}{$\begin{array}{c}\text { Bond Epitope Retrieval } \\
\text { Solution 2, (Leica Biosyste- } \\
\text { ms, Newcastle Upon Tyne, } \\
\text { UK)/20 min }\end{array}$} & \multirow{2}{*}{$\begin{array}{l}\text { Bond Polymer Refine } \\
\text { Detection System (Leica } \\
\text { Biosystems, Newcastle } \\
\text { Upon Tyne, UK), } 15 \text { min }\end{array}$} \\
\hline CK5/ XM26 & $\begin{array}{c}\text { Leica Biosystem Newcastle } \\
\text { Ltd, Newcastle Upon Tyne, } \\
\text { UK/15 min/RTU }\end{array}$ & & \\
\hline
\end{tabular}

counterstaining, were performed automatically on a Leica Bond-Max autostainer (Leica Biosystems, Newcastle Upon Tyne, UK) in accordance with the manufacturer's recommendations. The Her2 protocol was performed on Leica Bond Oracle Her2 IHC System. Modified Lille hematoxylin was used for nuclear counterstain.

\section{Microscopic evaluation}

The hormone receptor status was evaluated by using Allred score [12]. We combined the percentage of positive cells with intensity of nuclear staining from 10 microscopic fields. Cases with $+1-+3$ were considered ER, PR positive. 
The Her2 status was assessed according to ASCO recommendations [13]: "0" - no staining observed or weak, barely perceptible membrane staining until $10 \%$ of cells; " +1 " - weak membrane staining of $>$ $10 \%$; “+2" - incomplete, weak/moderate circumferential membrane staining $>10 \%$ of tumor cells or complete circumferential intense staining $<10 \%$ of cells; " +3 " - intense, circumferential staining of $>$ $10 \%$ of tumor cells. Cases with Her2 grade of +2 and +3 were considered as positive.

The CK5 expression was interpreted as Azoulay previously defined [14]: 0 - no tumor cells stained; +1 - less than $10 \%$ of tumor cells stained; $+2-$ $10-50 \%$ of positive tumor cells; $+3-$ more than $50 \%$ of tumor cells stained. Expression was scored positive $(>0)$ if any cytoplasmic and/or membranous staining tumor cells were observed.

For the Ki-67 marker we used a $14 \%$ threshold as the limit to define positive/negative cases [11]. The results were grouped in 7 subgroups:

1. $\mathrm{ER}^{-}, \mathrm{PR}^{-}, \mathrm{Her} 2^{-}, \mathrm{CK}^{-}$as $5 \mathrm{NP}$ (five negative phenotype),

2. $\mathrm{ER}^{-}, \mathrm{PR}^{-}, \mathrm{Her} 2^{-}, \mathrm{CK}^{+}{ }^{+}$as basal-like,

3. $\mathrm{ER}^{-}, \mathrm{PR}^{-}, \mathrm{Her} 2^{+}, \mathrm{CK}^{-}{ }^{-}$as Her2 ${ }^{+}$(Her2 overexpressed),

4. $\mathrm{ER}^{+}$and/or $\mathrm{PR}^{+}, \mathrm{Her}^{+}, \mathrm{CK}^{-}, \mathrm{Ki}-67<14$ as luminal $\mathrm{B} / \mathrm{Her} 2$,

Table II. The frequency of subtypes in primary tumors

\begin{tabular}{|c|c|c|c|c|}
\hline SuBtyPe & $\begin{array}{c}\text { No. OF } \\
\text { CASES }\end{array}$ & & $\%$ & \\
\hline $5 \mathrm{NP}$ & 2 & 4.65 & \multirow{2}{*}{11.63} & \multirow{2}{*}{11.63} \\
\hline Her2 & 3 & 6.98 & & \\
\hline Luminal A & 14 & 32.56 & 32.56 & \multirow{4}{*}{88.37} \\
\hline Luminal B/Her2 & 2 & 4.65 & \multirow{3}{*}{55.81} & \\
\hline Luminal B/Her2/Ki-67 & 5 & 11.63 & & \\
\hline Luminal B/Ki-67 & 17 & 39.53 & & \\
\hline Total & 43 & 100 & 100 & 100 \\
\hline
\end{tabular}

Table III. The frequency of subtypes at lymph node metastasis level (LNM)

\begin{tabular}{|c|c|c|c|c|}
\hline SUBTYPE & $\begin{array}{c}\text { No. OF } \\
\text { CASES }\end{array}$ & & $\%$ & \\
\hline $5 \mathrm{NP}$ & 3 & 6.98 & \multirow[t]{3}{*}{18.6} & \multirow[t]{3}{*}{18.6} \\
\hline Basal-like & 1 & 2.33 & & \\
\hline Her2 & 4 & 9.30 & & \\
\hline Luminal A & 16 & 37.21 & 37.21 & \multirow[t]{4}{*}{81.4} \\
\hline Luminal B/Her2 & 3 & 6.98 & \multirow[t]{3}{*}{44.19} & \\
\hline Luminal B/Her2/Ki-67 & 1 & 2.33 & & \\
\hline Luminal B/Ki-67 & 15 & 34.88 & & \\
\hline Total & 43 & 100 & 100 & 100 \\
\hline
\end{tabular}

5. $\mathrm{ER}^{+}$and/or $\mathrm{PR}^{+}, \mathrm{Her}^{+}, \mathrm{CK}^{-}, \mathrm{Ki}-67>14$ as luminal B/Her2/Ki-67,

6. $\mathrm{ER}^{+}$and/or $\mathrm{PR}^{+}, \mathrm{Her} 2^{-}, \mathrm{CK}^{-}, \mathrm{Ki}-67>14$ as luminal B/Ki-67,

7. $\mathrm{ER}^{+}$and/or $\mathrm{PR}^{+}, \mathrm{Her} 2^{-}, \mathrm{CK}^{-}, \mathrm{Ki}-67<14$ as luminal A.

\section{Image acquisition and data processing}

Three pathologists scored immunohistochemical results independently. The number of ER, PR, Ki-67 positive cells was evaluated on a Nikon Eclipse 80i instrument using a Nikon DS-Fil installed camera and Nis-elements 2.30 imaging software in accordance with Suciu's method [15]. The average from ten microscope fields $(40 \times)$ with the greatest number of positive cells was determined. A MS Access 2003 database was used to store and group the data.

\section{Statistical analysis}

The WINSTAT 2012.1 software was used for descriptive statistics. In order to determine the shifting direction (from basal to luminal and vice versa) and Pearson's correlation coefficient evaluation, $5 \mathrm{NP}$ subtype was assigned as "1", basal-like as " 2 ", Her2 $2^{+}$as "3", luminal B/Her2 as "4", luminal B/Ki-67 as "5", luminal B/Her2/Ki-67 as "6" and luminal A as "7". A p value of less than 0.05 was considered significant.

\section{Ethics}

The study has been approved by the Ethics Committee of the "Nicolae Testemitanu" University of Medicine and Pharmacy, Chisinau, Republic of Moldova, based on patients' informed consent.

\section{Results}

The luminal group was observed in the most of the cases (38 cases or $88.37 \%$ ) in comparison to the basal one ( 5 cases, $11.63 \%$ ) at the primary tumor level. Luminal B/Ki-67 was the most common subtype (39.53\%) followed by luminal A with $32.56 \%$ (Table II). The basal group consisted of $5 \mathrm{NP}$ and $\mathrm{Her}^{+}$ subtypes. None of the selected cases fulfilled the criteria of basal-like subtype.

In the lymph node metastasis the luminal group was also the most common -35 cases $/ 81.4 \%$ (Table III). Luminal B was considered as a leader of this group too $(44.19 \%)$, followed by luminal A with $37.21 \%$. In the structure of the basal group (18.6\%) one case of basal-like subtype was determined.

By comparing the molecular subtype of primary tumors and their metastases, we realized that in 10 cases $(23.26 \%)$ the subtype from lymph node metastasis shifted to another one (Table IV).

The statistical analysis reveals that the hormonal receptors' grade from tumor (ERtm, PRtm) correlates 
Table IV. Molecular subtypes defined by surrogate markers in primary tumors compared to their metastasis in axillary lymph nodes

\begin{tabular}{cccc}
\hline Primary Tumor subTYPe & LNM subTYPE & No. & $\%$ \\
\hline 5NP & 5 NP & 2 & 4.65 \\
\hline Her2 & Her2 & 3 & 6.98 \\
\hline Luminal A & Luminal A & 11 & 25.58 \\
\hline Luminal B/Her2 & Luminal B/Her2 & 1 & 2.33 \\
\hline Luminal B/Her2/Ki-67 & Luminal B/Her2 & 1 & 2.33 \\
\hline Luminal B/Her2/Ki-67 & Luminal B/Her2/Ki-67 & 1 & 2.33 \\
\hline Luminal B/Her2/Ki-67 & Luminal B/Ki-67 & 1 & 2.33 \\
\hline Luminal B/Ki-67 & Luminal B/Ki-67 & 13 & 30.23 \\
\hline Luminal A & 5 NP & 1 & 2.33 \\
\hline Luminal A & Luminal B/Her2 & 1 & 2.33 \\
\hline Luminal A & Luminal B/Ki-67 & 1 & 2.33 \\
\hline Luminal B/Her2 & Luminal A & 1 & 2.33 \\
\hline Luminal B/Her2/Ki-67 & Her2 & 1 & 2.33 \\
\hline Luminal B/Her2/Ki-67 & Luminal A & 1 & 2.33 \\
\hline Luminal B/Ki-67 & BasalLike & 1 & 2.33 \\
\hline Luminal B/Ki-67 & Luminal A & 3 & 6.98 \\
\hline
\end{tabular}

positively with homologous markers from metastasis and molecular subtypes from both sites (Table V). Increase of hormonal grade correlates negatively with Her2 and CK5 status. The level of proliferation marker Ki-67 from the primary tumor is linked with $\mathrm{Ki}-67$ and CK5 grade from metastasis. A negative correlation was determined between $\mathrm{Ki}-67$ value and tumor subtype.

\section{Discussion}

Using gene expression profiling, Weigelt et al. confirmed that human primary breast tumors are similar to distant metastases (in lung, ovary, skin, lymph node) of the same patient [16]. Van der Vijver also considers that the metastatic proficiency of a tumor is pre-programmed from its beginning and supports Bernards' results indicating that metastatic outcome is determined by events occurring early in the development of a tumor, rather than being dictated exclusively by events that occur many years later at the culmination of tumor progression $[17,18]$.

On the other hand, a series of data revealed differences in the expression signatures of tumors derived from cloned weakly/non-metastatic human cell lines and from their isogenic metastatic counterparts of the same patient $[19,20]$. This is in line with previous studies, which provided direct proof that individual malignant cells, co-existing within a given tumor, differ in metastatic capability [21]. Plus, as the metastatic ability of the cell population increases, the receptor profile changes concomitantly. This conclusively demonstrates that the molecular signature of breast carcinoma is not pre-determined and static, but continues to evolve in a tumor throughout its life history [7].

Nowadays, gene expression analysis has resulted in the definition of several different subtypes of breast cancer $[4,5]$. Because obtaining gene expression array information is quite laborious and expensive, Cheang et al. proposed a useful shorthand [22]. According to Goldhirsch A. this approach uses the immunohistochemical definition of estrogen and progesterone receptor to define the hormonal-dependent (or luminal) and hormonal-independent (or non-luminal) group [11]. Detection of human epidermal growth factor receptor- 2 overexpression was proposed as routine practice to uncover the $\mathrm{Her} 2^{+}$subtype. The $\mathrm{Ki}-67$ proliferation marker and Her2 stratify luminal $\mathrm{B}$, and $\mathrm{CK} 5$ reveals the basal-like subtype. In our research approach we tried to act from a usual clinical laboratory position and used all five surrogate markers to define the intrinsic subtypes of breast cancer.

As stated previously, the most common subtypes in breast carcinoma classification belong to the luminal group [23]. In our results as well, the luminal one was the richest, and luminal B had the majority part. As Ki-67 has a crucial role in defining the patients' future, we considered it useful to describe its activity in luminal $\mathrm{B} / \mathrm{Her} 2$ cases and subdivided it 
Table V. Spearman rank correlation between surrogate markers, Her2 status, molecular subtypes from primary tumor and lymph node metastasis (LNM)




into 2 subgroups, with low and high activity of proliferation (luminal B/Her2/Ki-67). This division allowed us to determine that these cases "like" to have a high proliferative activity (5 from 7 cases). Such results correlate with Cheang's data concerning high aggressiveness of luminal B/Her2 subtype [22]. But, 2 cases had a low Ki-67 index, so for other groups it is suggested to evaluate the prognostic value of luminal B/Her2 subclassification by Ki-67 level.

In our results, both hormone receptors (ER and PR) showed a strong similarity of correlations at both sites, the primary tumor and its metastasis. It is natural that both of them correlated positively with intrinsic subtype, as we associated the luminal group with the highest marks during statistical analysis. These markers look quite stable during metastasis. The single difference between hormone receptors was that only the level of the tumor's ER (without PR) correlated negatively with the basal marker CK5 from lymph node metastasis (LNM) (Table V).

All surrogate markers from our study correlated positively with their level from LNM. Such results could drive us to a hasty conclusion that cell profiles from both sites are homogeneous.

The most common determined "intrinsic" subtype at both sites in our assays was luminal B. But in 7 cases this subtype shifted to another one (Table IV). The highest frequency of changes was encountered from luminal $\mathrm{B} / \mathrm{Ki}-67$ to luminal $\mathrm{A}$ by diminishing the rate of proliferation. As we counted the $\mathrm{Ki}-67$ positive cells thoroughly, as the primary tumor and metastasis were on the same slide and technical risks were reduced to a minimum, we have to recognize that the panel of five enables one to describe the reason for this switch. These results can be explained partly by Zhou's data, where luminal A and B subtypes had the highest risk of metastasis in non-sentinel lymph nodes [24]. These 7 cases are in contradiction with Park's data, which affirm significant up-regulation of $\mathrm{Ki}-67$ protein in the metastatic site compared to the primary tumor [25].

The total number of switched cases was 10 from $43(23.26 \%)$. In five of them the subtype changed to one with a poor prognosis. The possible reasons could be different. Falck et al. also concluded that molecular profiles are not stable throughout tumor progression in breast cancer [10]. This supports the hypothesis that the malignant phenotype and its molecular signature are not pre-determined and static, but continue to evolve in a tumor throughout its life history [7].

On the other hand, Prat et al. noted that up to $10 \%$ of basal-like tumors are also positive for hormone receptors [5]. This genetic instability, when luminal receptors are determined immunohistochemically (IHC) in the basal one and vice versa, restricts the practical utility of surrogate markers. In our results one luminal $\mathrm{B} / \mathrm{Ki}-67$ subtype shifted to basal-like be- cause of +3 grade of CK5, which in Nielsen's opinion is driving the case in a group with significantly poorer outcome [26]. Similar results to ours, about migration to a poor prognosis subtype and increased aggressiveness of luminal and triple-negative subtypes throughout tumor progression, were reported by Castaneda [27].

There are some limitations of our study. We classified the tumors according to their ER, PR, Ki-67, CK5 and Her 2 status based on IHC surrogates, which is only an approximation of the genotype-based breast cancer subtype. But, nowadays IHC assays are cost-effective and have been accepted as useful clinical tests by many scientific communities.

According to Carey et al. the immunohistochemical definition of basal-like subtype is "ER negative, PR negative, Her 2 negative, cytokeratin $5 / 6$ positive and/or HER-1 positive" [28]. As we did not use the HER-1 marker, it is possible that some $5 \mathrm{NP}$ cases ( 2 in the primary tumor and 3 in LNM) in fact could be basal-like. However, the total amount of cases in the basal group did not change.

In the structure of the non-luminal basal group, oncologists recognized another molecular subtype, 5 negative phenotype (negative for all five markers, $5 \mathrm{NP}$ ), which is proven to be histologically less aggressive than basal-like and more aggressive than luminal A tumors [28, 29]. We encountered such a phenotype in 2 cases at the primary level and 3 in LNM. Could it be a "claudin-low" some of them we can't say, but a dangerous switch of luminal A to $5 \mathrm{NP}$ was determined once.

Her2 marker grade was increased in 4 from 10 switched cases. One has to recognize the fact that the lack of hybridization techniques in our tests could misclassify these cases. If to omit Her 2 involved cases, the rate of switched cases ( 4 from 43) remains high anyway and possibilities of shifting of one molecular subtype to another during the metastatic process remain a source for future debates.

In conclusion, our data support the hypothesis that breast cancer is a heterogeneous disease, with substantial variability of cellular components within each category, a statement applicable in invasive breast carcinomas of NST type too. The receptor profile of this carcinoma, indicated by surrogate markers, is not stable throughout the metastatic process.

The authors declare no conflict of interest.

This work was supported by UEFISCDI_Bilateral Cooperation Romania-Moldova grant 684/2013 of the Romanian Ministry of Education and Research and IDEI Research Grant 345/2011 of the Romanian Ministry of Education and Research. 


\section{References}

1. American Cancer Society. Global Cancer Facts \& Figures. $2^{\text {nd }}$ Edition, Atlanta 2011.

2. Huber KE, Carey LA, Wazer DE. Breast cancer molecular subtypes in patients with locally advanced disease: impact on prognosis, patterns of recurrence, and response to therapy. Semin Radiat Oncol 2009; 19: 204-210.

3. Zaha DC, Lazăr E, Lăzureanu C. Clinicopathologic features and five years survival analysis in molecular subtypes of breast cancer. Rom J Morphol Embryol 2010; 51: 85-89.

4. Perou CM, Sørlie T, Eisen MB, et al. Molecular portraits of human breast tumors. Nature 2000; 406: 747-752.

5. Prat A, Perou CM. Deconstructing the molecular portraits of breast cancer. Mol Oncol 2011; 5: 5-23.

6. Hao X, Sun B, Hu L, et al. Differential gene and protein expression in primary breast malignancies and their lymph node metastases as revealed by combined cDNA microarray and tissue microarray analysis. Cancer 2004; 100: 1110-1122.

7. Suzuki M, Tarin D. Gene expression profiling of human lymph node metastases and matched primary breast carcinomas: Clinical implications. Mol Oncol 2007; 1: 172-180.

8. Falck AK, Fernö M, Bendahl PO, et al. Does analysis of biomarkers in tumor cells in lymph node metastases give additional prognostic information in primary breast cancer? World J Surg 2010; 34: 1434-1441.

9. Falck AK, Ferno M, Bendahl PO, et al. St Gallen molecular subtypes in primary breast cancer and matched lymph node metastases - aspects on distribution and prognosis for patients with luminal A tumours: results from a prospective randomised trial. BMC Cancer 2013; 13: 558.

10. Falck AK, Bendahl PO, Chebil G, et al. Biomarker expression and St Gallen molecular subtype classification in primary tumours, synchronous lymph node metastases and asynchronous relapses in primary breast cancer patients with 10 years' follow-up. Breast Cancer Res Treat 2013; 140: 93-104.

11. Goldhirsch A, Wood WC, Coates AS, et al. Strategies for subtypes - dealing with the diversity of breast cancer: highlights of the St Gallen International Expert Consensus on the Primary Therapy of Early Breast Cancer. Ann Oncol 2011; 22: 1736-1747.

12. Allred DC, Harvey JM, Berardo M, et al. Prognostic and predictive factors in breast cancer by immunohistochemical analysis. Mod Pathol 1998; 11: 155-168.

13. Wolff AC, Hammond ME, Hicks DG, et al. Recommendations for Human Epidermal Growth Factor Receptor 2 Testing in Breast Cancer: American Society of Clinical Oncology/College of American Pathologists Clinical Practice Guideline Update. J Clin Oncol 2013; 31: 3997-4013.

14. Azoulay S, Laé M, Fréneaux P, et al. KIT is highly expressed in adenoid cystic carcinoma of the breast, a basal-like carcinoma associated with a favorable outcome. Mod Pathol 2005; 18 : 1623-1631.

15. Suciu C, Muresan AM, Cornea R, et al. Semi-automated evaluation of $\mathrm{Ki}-67$ index in invasive ductal carcinoma of the breast. Oncol Lett 2014; 7: 107-114.

16. Weigelt B., Glas AM, Lodewyk F, et al. Gene expression profiles of primary breast tumors maintained in distant metastases. Proc Natl Acad Sci U S A 2003; 100: 15901-15905.

17. van der Vijver MJ, He YD, van't Veer LJ, et al. A gene-expression signature as a predictor of survival in breast cancer $\mathrm{N}$ Engl J Med 2002; 347: 1999-2009.

18. Bernards R, Weinberg RA A progression puzzle. Nature 2002; 418: 823 .

19. Montel V, Huang TY, Mose E, et al. Expression profiling of primary tumors and matched lymphatic and lung metastases in a xenogeneic breast cancer model. Am J Pathol 2005; 166: 1565-1579.
20. Montel V, Mose ES, Tarin D. Tumor-stromal interactions reciprocally modulate gene expression patterns during carcinogenesis and metastasis. Int J Cancer 2006; 119: 251-263.

21. Urquidi V, Sloan D, Kawai K, et al. Contrasting expression of thrombospondin-1 and osteopontin correlates with absence or presence of metastatic phenotype in an isogenic model of spontaneous human breast cancer metastasis. Clin. Cancer Res 2002; 8: 61-74.

22. Cheang MC, Chia SK, Voduc D, et al. Ki67 index, HER2 status, and prognosis of patients with luminal B breast cancer. J Natl Cancer Inst 2009; 101: 736-750.

23. Engstrøm MJ, Opdahl S, Hagen AI, et al. Molecular subtypes, histopathological grade and survival in a historic cohort of breast cancer patients. Breast Cancer Res Treat 2013; 140: 463-473.

24. Zhou W, He Z, Xue J, et al. Molecular subtype classification is a determinant of non-sentinel lymph node metastasis in breast cancer patients with positive sentinel lymph nodes. PLoS One 2012; 7: e35881.

25. Park D, Kåresen R, Noren T, et al. Ki-67 expression in primary breast carcinomas and their axillary lymph node metastases: clinical implications. Virchows Arch 2007; 451: 11-18.

26. Nielsen TO, Hsu FD, Jensen $\mathrm{K}$, et al. Immunohistochemical and clinical characterization of the basal-like subtype of invasive breast carcinoma. Clin Cancer Res 2004; 10: 5367-5374.

27. Castaneda CA, Andrés E, Barcena C, et al. Behaviour of breast cancer molecular subtypes through tumour progression. Clin Transl Oncol 2012; 14: 481-485.

28. Carey LA, Perou CM, Livasy CA, et al. Race, breast cancer subtypes and survival in the Carolina breast cancer study. JAMA 2006; 295: 2492-2502.

29. Huo D, Ikpatt F, Khramtsov A, et al. Population differences in breast cancer: survey indigenous African women reveal over-representation of triple-negative breast cancer. J Clin Oncol 2009; 27: 4515-4521.

\section{Address for correspondence}

Anca Maria Cimpean, MD, PhD, Hab. Dr, Full Professor of Histology

Department of Microscopic Morphology/Histology

Angiogenesis Research Center

"Victor Babes" University of Medicine and Pharmacy

Piata Eftimie Murgu 2

300041, Timisoara, Romania

tel. +40720060955

e-mail: ancacimpean1972@yahoo.com, acimpeanu@umft.ro 